\title{
Bayesian Evidence on the Structure of Unemployment*
}

\author{
Peter M. Summers \\ Melbourne Institute of Applied Economic and Social Research \\ The University of Melbourne
}

Melbourne Institute Working Paper No. 3/03

ISSN 1328-4991 (Print)

ISSN 1447-5863 (Online)

ISBN 0734031157

February 2003

* Thanks to Don Harding and David Hendry for helpful discussions. I am very grateful
to David H. Papell for supplying me with his data set, and to Jiahui Wang and Eric
Zivot for sharing their Gauss code. Computations in this paper were carried out (in part)
using the Bayesian Analysis, Computation and Communications (BACC) software
available at www.econ.umn.edu/ bacc, and described in Geweke (1999). Any errors
are solely my responsibility. This research was partially supported by the Australian
Research Council under grant C00002131. Email address for correspondence:
p.summers@unimelb.edu.au

Melbourne Institute of Applied Economic and Social Research

The University of Melbourne

Victoria 3010 Australia

Telephone (03) 83443701

Fax (03) 83445630

Email melb-inst@unimelb.edu.au

WWW Address http://www.melbourneinstitute.com 


\begin{abstract}
This paper presents a Bayesian assessment of the likelihood of unit roots in the unemployment rates of 16 OECD countries. Bayesian techniques for detecting multiple structural breaks in time series have recently been developed by Wang and Zivot (2000). I apply these tests to a data set recently analyzed by Papell et al (2000). I also treat the number of structural breaks as an additional parameter to be estimated. I find virtually no support for unit root hysteresis in OECD unemployment rates; this result is very robust to the choice of prior.
\end{abstract}

JEL classification: C220, C110, E240

Keywords: multiple structural breaks; Bayesian analysis; unit root; unemployment rate 


\section{Introduction}

In a recent paper, Papell, Murray \& Ghiblawi (2000) (PMG hereafter) analyze the time series properties of unemployment rates in sixteen OECD countries. Their interest is in whether movements in these series are best described by so-called 'structuralist' theories, which imply that the unemployment rate is stationary about the natural rate, or by theories incorporating a unit root form of hysteresis, in which shocks to the unemployment rate persist forever. PMG subject the unemployment rates of the OECD countries to the unit root tests of Perron \& Vogelsang (1992) and Bai \& Perron (1998). These tests allow for the presence of structural breaks under the null hypothesis of a unit root. Allowing for structural breaks turns out to be crucial in this instance; when using standard augmented Dickey-Fuller (ADF) tests, PMG do not reject the unit root null for any of the 16 countries in their sample. Once allowance is made for structural breaks however, unit roots seem much less likely; the unit root null is rejected in ten countries. Further tests that allow for multiple breaks provide even more evidence for structuralist theories.

This paper presents a reassessment of PMG's results from a Bayesian perspective. As is well-known, the issue of inference about unit roots in time series models is on area in which Bayesian and frequentist methods can produce dramatically divergent conclusions. Sims \& Uhlig (1991), Uhlig (1994) and Bauwens, Lubrano \& Richard (1999) provide thorough discussions of how and why this divergence occurs. Bayesian techniques for detecting multiple structural breaks in the level, trend and variance of time series have recently been developed by Wang \& Zivot (2000). I analyze the same data as that used by PMG using the tests of Wang \& Zivot (2000), with the number of structural breaks treated as an additional parameter to be estimated. Using a range of priors for the autoregressive parameter in the model, I find virtually no support for unit root hysteresis in OECD unemployment rates. 


\section{The Model and Tests}

My analysis proceeds roughly in parallel with that of PMG. I first conduct unit root tests which do not allow for structural breaks. The general form of the model is

$$
A(L) u_{t}=\mu+\varepsilon_{t}
$$

where $u_{t}$ is the unemployment rate, $A(L)=1-a_{1} L-\cdots-a_{p} L^{p}$ is a $p$-th order lag polynomial, and $\varepsilon_{t} \sim N\left(0, \sigma^{2}\right)$. A unit root is present in (1) if $A(1)=0$. Following Lubrano (1995), I find it convenient to re-parameterize (1) as follows:

$$
u_{t}=\mu(1-\rho)+\rho u_{t-1}-A^{*}(L) \Delta u_{t}+\varepsilon_{t} .
$$

In (2), $\rho=1-A(1)$, and $A^{*}(L)$ is a $(p-1)$-th order polynomial with typical element given by:

$$
a_{j}^{*}=-\sum_{i=j+1}^{p} a_{i} .
$$

I chose $k$, the number of lagged difference terms, by a procedure similar to that of PMG. Beginning with $k=6$, I estimate (2) and check whether the last lagged difference term is significant. If not I reduce $k$ by one and re-estimate the model, continuing until either the last lag is significant or $k=0$. Here, 'significant' means that zero is not included in the $95 \%$ highest posterior density region for $a_{j}^{*}$.

For all the models considered here I use prior distributions which are centered on a random walk with no drift, and which imply a belief that the countries' unemployment rates are relatively homogeneous. In particular, the priors are: 


$$
\begin{aligned}
& \mu^{\sim} N(0,1000) \\
& \rho^{\sim} N(1,1000) \\
& a_{i}^{* \sim} N(0,1000) \forall i \\
& \sigma^{2 \sim} I G(3,1) .
\end{aligned}
$$

The inverted gamma $(I G)$ prior for $\sigma^{2}$ has a mean of 1 , while the average variance of a random walk model fitted to each of the 16 countries in the sample is about 0.96 . The priors are proper, but quite diffuse (unrealistically so in the case of $\rho$; this is discussed further below). Because I use Bayes factors and posterior odds ratios to discriminate between competing models, it is necessary to use proper priors in order to ensure that these quantities are well-defined.

\subsection{Unit root priors}

As pointed out by Lubrano (1995), Bauwens, Lubrano \& Richard (1999), and Uhlig (1994), specification of the prior on $\rho$ requires particular care, especially regarding the treatment of explosive values. The Normal prior as specified above may be regarded as an unsuitable prior for time series with possible unit roots, as it puts more weight on the explosive region (including $\rho>1$ and $\rho<-1$ ) than the stationary region. Nevertheless, Uhlig (1994) argues that a Normal prior centred on a random walk is 'reasonable' if there is no time trend in the model (as is the case here). Therefore I use this prior as a convenient base case, but also conduct inference using four other priors that are arguably more realistic. The first of these simply tightens the variance in the base prior from 1,000 to 0.5 . The second follows an idea of Lubrano (1995) and Bauwens, Lubrano \& Richard (1999), and is a Beta

prior extended to the range $-\sqrt{1+v} \leq \rho \leq \sqrt{1+v}$. I set $v=0.5$, which restricts $\rho$ to 
the interval $[-1.225,1.225]$. This value of $v$ also favors the non-stationary region by about two-to-one. The fourth prior is a $\operatorname{Beta}(10,2)$ distribution on the same extended range as for Lubrano's prior. This prior has a mean of 0.82 , standard deviation of 0.25 , and a mode at 0.98. Although it favors roots near unity, this prior also makes explosive roots much less likely than Lubrano's prior. The final prior is that of Berger \& Yang (1994), extended to the non-stationary region. This prior is given by

$$
f(\rho)=\left\{\begin{array}{c}
\left(2 \pi \sqrt{1-\rho^{2}}\right)^{-1},|\rho|<1 \\
\left(2 \pi|\rho| \sqrt{\rho^{2}-1}\right)^{-1},|\rho| \geq 1 .
\end{array}\right.
$$

See Bauwens, Lubrano \& Richard (1999), p. 177 for more details of this prior. Figure 1 graphs the various priors on the interval $\rho \in[0.5,1.2]$ (the $N(1,1000)$ prior is not shown as it would be indistinguishable from the $\mathrm{x}$-axis).

I investigate the role of these prior distributions on unit root inference by using the reweighting method described in Geweke (1999). Suppose we have a sequence of $K$ draws of $\rho,\left\{\rho^{i}\right\}_{i=1}^{K}$ from the posterior $f_{N}(\rho \mid y)$ implied by the $N(1,1000)$ prior. Draws from the posterior implied by a different prior $f_{j}(\rho \mid y)$ can be obtained by simply multiplying the $\rho^{i}$ by the ratio of the new prior density to that of the $N(1,1000)$. That is,

$$
\left\{\rho_{j}^{i}\right\}_{i=1}^{K}=\left\{\rho^{i} \frac{f_{j}(\rho)}{f_{N}(\rho)}\right\}
$$

where $f_{j}(\rho)$ is the one of the alternative prior densities mentioned above.

\subsection{Bayesian inference in the multiple break model}

Wang \& Zivot (2000) have developed Bayesian methods for analyzing time series with an unknown number of breaks in the mean, trend, and/or variance. Consider a (first-order) version of equation (1) with $n$ structural breaks in the intercept: 


$$
u_{t}=\mu_{1}+\mu_{2}+\cdots+\mu_{n+1}+\rho u_{t-1}+\varepsilon_{t} .
$$

Defining $I_{A}$ to be the indicator function that takes the value 1 if event $A$ is true, Wang and Zivot write (3) as a linear regression (conditional on $n$ ) with $n+1$ dummy variables:

$$
u_{t}=\mu_{1} I_{\left(1 \leq t<k_{1}\right)}+\mu_{2} I_{\left(k_{1} \leq t<k_{2}\right)}+\cdots+\mu_{k+1} I_{\left(k_{n} \leq t<T\right)}+\rho u_{t-1}+\varepsilon_{t},
$$

with $k_{j}$ the time index of the $j^{\text {th }}$ break and $T$ the last observation in the sample. Thus, conditional on $n$, Bayesian inference in this model is straightforward using natural conjugate priors on the $\mu_{j}$ and $\sigma^{2}$, the residual variance.

To conduct inference on the locations of the $n$ breaks $k_{1}, k_{2}, \ldots, k_{n}$, Wang and Zivot specify a discrete prior that is uniform over all ordered subsequences of the time indexes $\{2,3, \ldots, T\}$ of length $n$. For example, in a model with 50 observations and 3 breaks, it is equally likely a priori that the breaks occur at times $\{5,12,37\},\{11,24,29\}$, or $\{2,3,4\}$. Note that in this last sequence, the first two 'breaks' can be thought of as additive outliers. Papell, Murray \& Ghiblawi (2000) require at least $15 \%$ of the sample to lie between any two break dates, whereas I leave the ordering of break dates unrestricted. Wang \& Zivot (2000) then show that the conditional posterior of the $j^{\text {th }}$ break $k_{j}$ depends only on its neighbors $k_{j-1}$ and $k_{j+1}$. Suppose that the interval $\left[k_{j-1}, k_{j+1}\right]$ contains $m$ points. The conditional posterior for $k_{j}$ is then multinomial: $k_{j} \sim M N\left(m, l^{m}\right)$, where $l^{m}=\left(l_{1}, l_{2}, \ldots, l_{m}\right)$ is the vector obtained by evaluating the likelihood function at each of the $m$ possible dates for $k_{j}$, properly normalized. See Wang \& Zivot (2000) for more details, including extensions to models with a trend term and regime-specific variances, as well as their Gibbs sampling algorithm. 


\subsection{Inference on the number of breaks}

All of the results in Wang \& Zivot (2000) are conditional on the number of break points, $n$. Those authors use the Bayesian Information Criterion (BIC) to choose among models with different values of $n$. In this paper, I take a different approach and treat $n$ as an additional parameter to be estimated. To do this I draw on recent results of Carlin \& Chib (1995), Han \& Carlin (2001), Godsill (2001), Dellaportas, Forster \& Ntzoufras (2002), and Lopes $\&$ West (2000). These papers describe Markov Chain Monte Carlo (MCMC) methods for conducting inference in situations involving competing models of varying dimension. My approach in this paper is closest to that of Lopes \& West (2000), who use a 'Metropolized' version of Carlin and Chib's method. The algorithm proceeds as follows:

1. Specify an initial number of breaks $n_{0} \in\left[0, n_{\max }\right]$, where $n_{\max }$ is the maximum number of breaks under consideration;

2. Draw a parameter vector $\theta_{0}=\left(k_{1}, \cdots, k_{n_{0}}, \mu_{1}, \cdots, \mu_{n+1}, \rho, \sigma^{2}\right)$ corresponding to the $n_{0}$-break model;

3. Propose a new number of breaks $n_{1}$ from a proposal distribution $J\left(n_{1}, n_{0}\right)$. In general, the proposed number of breaks, $n_{1}$, can depend on the current number of breaks, $n_{0}$.

4. Draw the corresponding parameter vector $\theta_{1}$ from a proposal distribution $q\left(\theta_{1}\right)$.

5. Accept the proposed move with probability

$$
\alpha=\min \left\{1, \frac{l_{1}\left(y \mid n_{1}, \theta_{1}\right)}{l_{0}\left(y \mid n_{0}, \theta_{0}\right)} \cdot \frac{\pi\left(\theta_{1} \mid n_{1}\right) \pi\left(n_{1}\right)}{\pi\left(\theta_{0} \mid n_{0}\right) \pi\left(n_{0}\right)} \cdot \frac{q\left(\theta_{0}\right)}{q\left(\theta_{1}\right)} \cdot \frac{J\left(n_{1}, n_{0}\right)}{J\left(n_{0}, n_{1}\right)}\right\}
$$

(the terms in this expression are discussed below);

6. If the move is accepted, set $n_{0}=n_{1}$; otherwise keep $n_{0}$ unchanged; 


\section{Go to 2 .}

This algorithm is referred to as 'Metropolized Carlin \& Chib' (MCC) by Dellaportas, Forster \& Ntzoufras (2002). Lopes \& West (2000) suggest that a more descriptive term is independence reversible jump MCMC, as it is a special case of the reversible jump algorithm of Green (1995). The first three terms in the acceptance probability $\alpha$ are: the ratio of the likelihood function values for the two models; the ratio of the prior distributions for the two parameter vectors (note that this is the ratio of the joint priors $\pi\left(\theta_{j}, n_{j}\right)$ ), and the ratio of the two proposal densities. The last term gives the ratio of the transition probabilities for moves between models with $n_{0}$ and $n_{1}$ breaks. In this paper, I make two assumptions that simplify the calculation of $\alpha$. First, I assume that all models with $n \in$ $[0,5]$ are equally a priori, so the $\pi\left(n_{j}\right)$ terms cancel (the choice of a maximum of five breaks follows that of Papell, Murray \& Ghiblawi (2000)). I also use a uniform proposal density for $J(.,$.$) , so the final term is equal to unity. This also means that n_{1}$ is independent of $n_{0}$. For the proposal distribution $q\left(\theta_{j}\right)$ I use the full conditional posteriors derived in Wang \& Zivot (2000): multinomial for the location of the breaks, Normal for the $\mu_{j}$ and $\rho$, and inverted Gamma for the variance $\sigma^{2}$. An alternative method would be to use the estimated posterior distributions from preliminary model-specific runs. This is the approach taken by Lopes \& West (2000).

As a preliminary step, I estimated models with zero to five breaks in the intercept term only. For each of these models, I ran the Gibbs sampler of Wang \& Zivot (2000) for 12,500 draws and omitted the first 2,500. The data are the same as in Papell, Murray \& Ghiblawi (2000), and consist of the annual average unemployment rates for 16 OECD countries. I then ran the independence reversible jump algorithm for 11,000 iterations, keeping the last 10,000. The posterior probabilities of the six models can then be estimated by the frequency of each model's occurrence in these 10,000 draws. Finally, I base my inference on the autoregressive parameter $\rho$ on the output of the preliminary runs, using the estimated 
posterior model probabilities as weights. Although the primary focus of this paper is the presence or absence of a unit root, a similar procedure can be used to average the posterior distributions of the break dates.

\section{Results}

\subsection{The number of structural breaks}

Table 1 gives the estimated posterior probabilities for each of the six models for each country. The last column of the table shows the percentage of proposed moves that were accepted. The results in the table differ substantially from those of Papell, Murray \& Ghiblawi (2000), who use the tests of Bai \& Perron (1998) (see their table 3). They find one significant structural break in Belgium, Norway and Sweden; two breaks in Canada, Denmark, Finland, Ireland, the UK and the US; and three breaks in Spain. The Bai-Perron tests are not applicable to the other countries since the unit root null cannot be rejected. The modal values in table 1 correspond to their results only for Norway. In addition, Papell, Murray \& Ghiblawi (2000) prefer a two-break model for Australia, although the unit root null is not rejected. My results suggest that the no-break and one-break models are about equally likely (and each about twice as likely as the two-break model). The acceptance probabilities $\hat{\alpha}$ range from 0.31 for Finland to 0.87 for Japan. On average for all countries, just over half of the proposed moves were accepted.

\subsection{Unit root inference}

Several features of the posterior distributions of $\rho$ are shown in table 2. In addition to the posterior mean, standard deviation and median, the table gives estimates of the tail areas of each distribution to the right of $\rho=0.90,0.95,0.975$ and 1 . I estimated the posterior densities using the 'smooth' command in BACC with a uniform kernel, then computed 
the tail areas using the midpoint of the $y$-ordinates of the resulting ordered pairs. The unemployment rates in 13 of the 16 countries are clearly stationary, although in some cases quite persistent. France, Italy and Spain are the only countries for which the posterior distribution of $\rho$ has appreciable mass above 0.975 . The only country for which there is clear evidence for a unit root is France. The median of $\rho$ is around 0.98 , and roughly onequarter of each of the various posterior distributions lies to the right of unity. The evidence for non-stationarity in the Italian and Spanish unemployment rates is much weaker. Unless one adopts the Berger-Yang prior, one would not reject the hypothesis that $\rho$ was at most 0.975 for these countries at conventional significance levels. For all of the other countries and all priors, at least 94 per cent of the posterior distribution of $\rho$ lies to the left of 0.95 .

\subsection{Unemployment persistence}

The posterior means of $\rho$ under the various priors are collected in table 3. Once allowance for structural breaks is made, the persistence of the unemployment rate across the various countries is remarkably similar. The last two columns of table 3 present estimates of the half-life of shocks to the unemployment rate. The first is computed using estimates of $\rho$ from an ADF-type regression, while the second uses the maximum value of the posterior means in the table. Most of these half-lives are under three years, and only three exceed five years.

\section{Discussion and Conclusions}

This paper strengthens the results of Papell, Murray \& Ghiblawi (2000) by adopting Bayesian inferential procedures that allow for model uncertainty as well as parameter uncertainty. These results provide strong support for the intuition that since the unemployment rate is a bounded series, it cannot have a unit root. The degree of persistence in the unemploy- 
ment rates of these countries is remarkably similar once allowance is made for an unknown number of structural breaks. One interpretation of this result is that differences in social welfare systems, minimum wage laws and so on may account for variation in the average level of unemployment rates, but do not affect their dynamic properties.

\section{References}

Bai, Jushan \& Pierre Perron. 1998. "Estimating and Testing Linear Models with Multiple Structural Changes." Econometrica 66(1):47-78.

Bauwens, Luc, Michel Lubrano \& Jean-Francois Richard. 1999. Bayesian Inference in Dynamic Econometric Models. Oxford: Oxford University Press.

Berger, James O. \& Ruo-Yong Yang. 1994. "Non-Informative Priors and Bayesian Testing for the AR(1) Model." Econometric Theory 10:461-482.

Carlin, Bradley P. \& Siddhartha Chib. 1995. "Bayesian Model Choice Via Markov Chain Monte Carlo." Journal of the Royal Statistical Society (Series B) 57(3):473-484.

Dellaportas, Petros, Jonathan J. Forster \& Ioannis Ntzoufras. 2002. “On Bayesian Model and Variable Selection Using MCMC." Statistics and Computing 12:27-36.

Geweke, John. 1999. "Using Simulation Methods for Bayesian Econometric Models: Inference, Development and Communication.” Econometric Reviews 18(1):1-73.

Godsill, Simon J. 2001. "On the Relationship Between Markov Chain Monte Carlo Methods for Model Uncertainty.” Journal of Computational and Graphical Statistics 10(2):230-248. 
Green, Peter J. 1995. "Reversible Jump Markov Chain Monte Carlo Computation and Bayesian Model Determination.” Biometrika 82(4):711-732.

Han, Cong \& Bradley P. Carlin. 2001. "Markov Chain Monte Carlo Methods for Computing Bayes Factors: A Comparative Review." Journal of the American Statistical Association 96(455):1122-1132.

Lopes, Hedibert F. \& Mike West. 2000. "Model Uncertainty in Factor Analysis." mimeo, Duke University .

Lubrano, Michel. 1995. “Testing for Unit Roots in a Bayesian Framework.” Journal of Econometrics 69:81-109.

Papell, David H., Christian J. Murray \& Hala Ghiblawi. 2000. “The Structure of Unemployment." Review of Economics and Statistics 82(2):309-315.

Perron, Pierre \& Timothy Vogelsang. 1992. "Nonstationarity and Level Shifts with an Application to Purchasing Power Parity." Journal of Business and Economic Statistics $10: 301-320$.

Sims, Christopher \& Harald Uhlig. 1991. "Understanding Unit Rooters: A Helicopter Tour." Econometrica 59:1591-1600.

Uhlig, Harald. 1994. "What Macroeconomists Should Know About Unit Roots: A Bayesian Perspective.” Econometric Theory 10:645-671.

Wang, Jiahui \& Eric Zivot. 2000. "A Bayesian Time Series Model of Multiple Structural Changes in Level, Trend, and Variance.” Journal of Business and Economic Statistics 18(3):374-386. 
Table 1: Posterior model probabilities

\begin{tabular}{|c|c|c|c|c|c|c|c|}
\hline & \multicolumn{6}{|c|}{ number of breaks } & \multirow[b]{2}{*}{$\hat{\alpha}$} \\
\hline & 0 & 1 & 2 & 3 & 4 & 5 & \\
\hline Australia & 31.69 & 32.09 & 16.45 & 9.35 & 5.49 & 4.93 & 0.53 \\
\hline Belgium & 10.28 & 12.46 & 15.8 & 8.17 & 22.91 & 30.38 & 0.52 \\
\hline Canada & 28.04 & 27.49 & 11.99 & 10.17 & 15.2 & 7.11 & 0.55 \\
\hline Denmark & 11.89 & 19.48 & 14.31 & 16.06 & 22.71 & 15.55 & 0.65 \\
\hline Finland & 3.33 & 5.93 & 7.51 & 12.01 & 33.65 & 37.57 & 0.31 \\
\hline France & 40.34 & 37.29 & 13.36 & 6.25 & 1.11 & 1.65 & 0.50 \\
\hline Germany & 30.64 & 25.13 & 11.3 & 5.77 & 14.64 & 12.52 & 0.41 \\
\hline Ireland & 17.2 & 29 & 12.33 & 10.17 & 23.26 & 8.04 & 0.49 \\
\hline Italy & 28.85 & 34.71 & 12.51 & 7.17 & 6.64 & 10.12 & 0.51 \\
\hline Japan & 19.4 & 25.95 & 18.95 & 18.06 & 12.99 & 4.65 & 0.87 \\
\hline Netherlands & 14.05 & 18.25 & 5.82 & 4.02 & 39.78 & 18.07 & 0.43 \\
\hline Norway & 29.7 & 28.67 & 17.22 & 14.99 & 8.86 & 0.56 & 0.58 \\
\hline Spain & 10.01 & 26.22 & 16.73 & 14.39 & 19.24 & 13.41 & 0.58 \\
\hline Sweden & 2.21 & 5.81 & 7.12 & 13.71 & 39.76 & 31.39 & 0.36 \\
\hline UK & 7.8 & 12.9 & 9.33 & 16.7 & 31.45 & 21.82 & 0.51 \\
\hline US & 25.97 & 28.6 & 16.15 & 11.27 & 11.41 & 6.6 & 0.56 \\
\hline
\end{tabular}


Table 2: Quantiles and tail areas of posterior distributions of $\rho$, various priors

\begin{tabular}{|c|c|c|c|c|c|}
\hline \multirow[b]{2}{*}{ Quantile } & \multicolumn{5}{|c|}{ Prior } \\
\hline & $N(1,1000)$ & $N(1,0.5)$ & Lubrano & $\beta(10,2)$ & Berger-Yang \\
\hline & \multicolumn{5}{|c|}{ Australia } \\
\hline mean & 0.75 & 0.75 & 0.75 & 0.76 & 0.76 \\
\hline $\mathrm{sd}$ & 0.05 & 0.05 & 0.05 & 0.05 & 0.05 \\
\hline median & 0.76 & 0.76 & 0.76 & 0.76 & 0.77 \\
\hline 0.90 & 0.00 & 0.00 & 0.00 & 0.00 & 0.00 \\
\hline 0.95 & 0.00 & 0.00 & 0.00 & 0.00 & 0.00 \\
\hline 0.975 & 0.00 & 0.00 & 0.00 & 0.00 & 0.00 \\
\hline \multirow[t]{2}{*}{1} & 0.00 & 0.00 & 0.00 & 0.00 & 0.00 \\
\hline & \multicolumn{5}{|c|}{ Belgium } \\
\hline mean & 0.67 & 0.67 & 0.67 & 0.68 & 0.67 \\
\hline sd & 0.04 & 0.04 & 0.04 & 0.04 & 0.04 \\
\hline median & 0.68 & 0.68 & 0.68 & 0.68 & 0.68 \\
\hline 0.90 & 0.00 & 0.00 & 0.00 & 0.00 & 0.00 \\
\hline 0.95 & 0.00 & 0.00 & 0.00 & 0.00 & 0.00 \\
\hline 0.975 & 0.00 & 0.00 & 0.00 & 0.00 & 0.00 \\
\hline \multirow[t]{2}{*}{1} & 0.00 & 0.00 & 0.00 & 0.00 & 0.00 \\
\hline & \multicolumn{5}{|c|}{ Canada } \\
\hline mean & 0.78 & 0.78 & 0.78 & 0.78 & 0.78 \\
\hline $\mathrm{sd}$ & 0.05 & 0.05 & 0.05 & 0.05 & 0.05 \\
\hline median & 0.78 & 0.79 & 0.79 & 0.79 & 0.79 \\
\hline 0.90 & 0.01 & 0.01 & 0.01 & 0.01 & 0.01 \\
\hline 0.95 & 0.00 & 0.00 & 0.00 & 0.00 & 0.00 \\
\hline 0.975 & 0.00 & 0.00 & 0.00 & 0.00 & 0.00 \\
\hline \multirow[t]{2}{*}{1} & 0.00 & 0.00 & 0.00 & 0.00 & 0.00 \\
\hline & \multicolumn{5}{|c|}{ Denmark } \\
\hline mean & 0.73 & 0.73 & 0.73 & 0.74 & 0.74 \\
\hline sd & 0.07 & 0.07 & 0.07 & 0.07 & 0.07 \\
\hline median & 0.72 & 0.72 & 0.73 & 0.74 & 0.74 \\
\hline 0.90 & 0.01 & 0.01 & 0.01 & 0.01 & 0.01 \\
\hline 0.95 & 0.00 & 0.00 & 0.00 & 0.00 & 0.00 \\
\hline 0.975 & 0.00 & 0.00 & 0.00 & 0.00 & 0.00 \\
\hline 1 & 0.00 & 0.00 & 0.00 & 0.00 & 0.00 \\
\hline
\end{tabular}


Table 2: continued

\begin{tabular}{|c|c|c|c|c|c|}
\hline \multirow[b]{2}{*}{ Quantile } & \multicolumn{5}{|c|}{ Prior } \\
\hline & $N(1,1000)$ & $N(1,0.5)$ & Lubrano & $\beta(10,2)$ & Berger-Yang \\
\hline & \multicolumn{5}{|c|}{ Finland } \\
\hline mean & 0.64 & 0.65 & 0.65 & 0.69 & 0.68 \\
\hline sd & 0.14 & 0.13 & 0.14 & 0.13 & 0.15 \\
\hline median & 0.66 & 0.67 & 0.68 & 0.70 & 0.67 \\
\hline 0.90 & 0.04 & 0.04 & 0.05 & 0.06 & 0.10 \\
\hline 0.95 & 0.02 & 0.02 & 0.02 & 0.03 & 0.06 \\
\hline 0.975 & 0.01 & 0.01 & 0.02 & 0.02 & 0.04 \\
\hline \multirow[t]{2}{*}{1} & 0.01 & 0.01 & 0.01 & 0.01 & 0.03 \\
\hline & \multicolumn{5}{|c|}{ France } \\
\hline mean & 0.98 & 0.98 & 0.98 & 0.98 & 0.99 \\
\hline $\mathrm{sd}$ & 0.03 & 0.03 & 0.03 & 0.03 & 0.03 \\
\hline median & 0.98 & 0.98 & 0.99 & 0.98 & 0.98 \\
\hline 0.90 & 0.99 & 0.99 & 0.99 & 0.99 & 1.00 \\
\hline 0.95 & 0.76 & 0.76 & 0.77 & 0.76 & 0.87 \\
\hline 0.975 & 0.48 & 0.48 & 0.50 & 0.48 & 0.67 \\
\hline \multirow[t]{2}{*}{1} & 0.24 & 0.24 & 0.26 & 0.24 & 0.32 \\
\hline & \multicolumn{5}{|c|}{ Germany } \\
\hline mean & 0.80 & 0.80 & 0.80 & 0.80 & 0.80 \\
\hline sd & 0.05 & 0.05 & 0.05 & 0.05 & 0.05 \\
\hline median & 0.79 & 0.79 & 0.79 & 0.79 & 0.79 \\
\hline 0.90 & 0.01 & 0.01 & 0.02 & 0.02 & 0.02 \\
\hline 0.95 & 0.00 & 0.00 & 0.00 & 0.00 & 0.00 \\
\hline 0.975 & 0.00 & 0.00 & 0.00 & 0.00 & 0.00 \\
\hline \multirow[t]{2}{*}{1} & 0.00 & 0.00 & 0.00 & 0.00 & 0.00 \\
\hline & \multicolumn{5}{|c|}{ Ireland } \\
\hline mean & 0.76 & 0.76 & 0.76 & 0.76 & 0.76 \\
\hline sd & 0.05 & 0.05 & 0.05 & 0.05 & 0.05 \\
\hline median & 0.77 & 0.77 & 0.78 & 0.78 & 0.78 \\
\hline 0.90 & 0.00 & 0.00 & 0.00 & 0.00 & 0.01 \\
\hline 0.95 & 0.00 & 0.00 & 0.00 & 0.00 & 0.00 \\
\hline 0.975 & 0.00 & 0.00 & 0.00 & 0.00 & 0.00 \\
\hline 1 & 0.00 & 0.00 & 0.00 & 0.00 & 0.00 \\
\hline
\end{tabular}


Table 2: continued

\begin{tabular}{|c|c|c|c|c|c|}
\hline \multirow[b]{2}{*}{ Quantile } & \multicolumn{5}{|c|}{ Prior } \\
\hline & $\overline{N(1,1000)}$ & $N(1,0.5)$ & Lubrano & $\beta(10,2)$ & Berger-Yang \\
\hline & \multicolumn{5}{|c|}{ Italy } \\
\hline mean & 0.90 & 0.90 & 0.90 & 0.90 & 0.91 \\
\hline $\mathrm{sd}$ & 0.04 & 0.04 & 0.04 & 0.04 & 0.04 \\
\hline median & 0.89 & 0.90 & 0.90 & 0.90 & 0.90 \\
\hline 0.90 & 0.50 & 0.51 & 0.52 & 0.52 & 0.60 \\
\hline 0.95 & 0.12 & 0.13 & 0.14 & 0.13 & 0.21 \\
\hline 0.975 & 0.04 & 0.04 & 0.04 & 0.04 & 0.09 \\
\hline \multirow[t]{2}{*}{1} & 0.01 & 0.01 & 0.01 & 0.01 & 0.03 \\
\hline & \multicolumn{5}{|c|}{ Japan } \\
\hline mean & 0.83 & 0.83 & 0.84 & 0.84 & 0.84 \\
\hline sd & 0.05 & 0.05 & 0.05 & 0.05 & 0.05 \\
\hline median & 0.83 & 0.83 & 0.83 & 0.83 & 0.84 \\
\hline 0.90 & 0.10 & 0.10 & 0.11 & 0.11 & 0.13 \\
\hline 0.95 & 0.01 & 0.01 & 0.01 & 0.01 & 0.02 \\
\hline 0.975 & 0.00 & 0.00 & 0.00 & 0.00 & 0.01 \\
\hline \multirow[t]{2}{*}{1} & 0.00 & 0.00 & 0.00 & 0.00 & 0.00 \\
\hline & \multicolumn{5}{|c|}{ Netherlands } \\
\hline mean & 0.73 & 0.74 & 0.74 & 0.75 & 0.75 \\
\hline sd & 0.08 & 0.08 & 0.08 & 0.08 & 0.08 \\
\hline median & 0.75 & 0.75 & 0.75 & 0.76 & 0.76 \\
\hline 0.90 & 0.03 & 0.03 & 0.04 & 0.04 & 0.05 \\
\hline 0.95 & 0.00 & 0.00 & 0.00 & 0.00 & 0.01 \\
\hline 0.975 & 0.00 & 0.00 & 0.00 & 0.00 & 0.00 \\
\hline \multirow[t]{2}{*}{1} & 0.00 & 0.00 & 0.00 & 0.00 & 0.00 \\
\hline & \multicolumn{5}{|c|}{ Norway } \\
\hline mean & 0.63 & 0.64 & 0.64 & 0.64 & 0.64 \\
\hline sd & 0.05 & 0.05 & 0.05 & 0.05 & 0.05 \\
\hline median & 0.63 & 0.63 & 0.63 & 0.64 & 0.63 \\
\hline 0.90 & 0.00 & 0.00 & 0.00 & 0.00 & 0.00 \\
\hline 0.95 & 0.00 & 0.00 & 0.00 & 0.00 & 0.00 \\
\hline 0.975 & 0.00 & 0.00 & 0.00 & 0.00 & 0.00 \\
\hline 1 & 0.00 & 0.00 & 0.00 & 0.00 & 0.00 \\
\hline
\end{tabular}


Table 2: continued

\begin{tabular}{|c|c|c|c|c|c|}
\hline \multirow[b]{2}{*}{ Quantile } & \multicolumn{5}{|c|}{ Prior } \\
\hline & $N(1,1000)$ & $N(1,0.5)$ & Lubrano & $\beta(10,2)$ & Berger-Yang \\
\hline & \multicolumn{5}{|c|}{ Spain } \\
\hline mean & 0.87 & 0.87 & 0.88 & 0.88 & 0.89 \\
\hline sd & 0.04 & 0.04 & 0.04 & 0.04 & 0.05 \\
\hline median & 0.90 & 0.90 & 0.90 & 0.90 & 0.90 \\
\hline 0.90 & 0.25 & 0.25 & 0.27 & 0.26 & 0.35 \\
\hline 0.95 & 0.06 & 0.06 & 0.07 & 0.07 & 0.14 \\
\hline 0.975 & 0.03 & 0.03 & 0.03 & 0.03 & 0.08 \\
\hline \multirow[t]{2}{*}{1} & 0.01 & 0.01 & 0.01 & 0.01 & 0.04 \\
\hline & \multicolumn{5}{|c|}{ Sweden } \\
\hline mean & 0.59 & 0.61 & 0.61 & 0.67 & 0.62 \\
\hline sd & 0.16 & 0.15 & 0.16 & 0.14 & 0.16 \\
\hline median & 0.55 & 0.57 & 0.56 & 0.60 & 0.57 \\
\hline 0.90 & 0.02 & 0.03 & 0.03 & 0.04 & 0.04 \\
\hline 0.95 & 0.01 & 0.01 & 0.01 & 0.01 & 0.02 \\
\hline 0.975 & 0.00 & 0.00 & 0.00 & 0.00 & 0.01 \\
\hline \multirow[t]{2}{*}{1} & 0.00 & 0.00 & 0.00 & 0.00 & 0.00 \\
\hline & \multicolumn{5}{|c|}{ UK } \\
\hline mean & 0.60 & 0.61 & 0.61 & 0.62 & 0.61 \\
\hline $\mathrm{sd}$ & 0.07 & 0.07 & 0.07 & 0.07 & 0.07 \\
\hline median & 0.63 & 0.64 & 0.64 & 0.65 & 0.64 \\
\hline 0.90 & 0.00 & 0.00 & 0.00 & 0.00 & 0.00 \\
\hline 0.95 & 0.00 & 0.00 & 0.00 & 0.00 & 0.00 \\
\hline 0.975 & 0.00 & 0.00 & 0.00 & 0.00 & 0.00 \\
\hline \multirow[t]{2}{*}{1} & 0.00 & 0.00 & 0.00 & 0.00 & 0.00 \\
\hline & \multicolumn{5}{|c|}{ US } \\
\hline mean & 0.67 & 0.67 & 0.67 & 0.68 & 0.68 \\
\hline sd & 0.06 & 0.06 & 0.06 & 0.06 & 0.06 \\
\hline median & 0.66 & 0.67 & 0.67 & 0.67 & 0.67 \\
\hline 0.90 & 0.00 & 0.00 & 0.00 & 0.00 & 0.00 \\
\hline 0.95 & 0.00 & 0.00 & 0.00 & 0.00 & 0.00 \\
\hline 0.975 & 0.00 & 0.00 & 0.00 & 0.00 & 0.00 \\
\hline 1 & 0.00 & 0.00 & 0.00 & 0.00 & 0.00 \\
\hline
\end{tabular}


Table 3: Posterior means of $\rho$ and half-lives

\begin{tabular}{rrrrrrrr}
\hline \hline & $\mathrm{N}(1,1000)$ & $\mathrm{N}(1,0.5)$ & Lubrano & $\beta(10,2)$ & $\mathrm{B}-\mathrm{Y}$ & \multicolumn{2}{c}{ half-life } \\
& & & & & & ADF & max \\
\hline Australia & 0.75 & 0.75 & 0.75 & 0.76 & 0.76 & 13.05 & 2.51 \\
Belgium & 0.67 & 0.67 & 0.67 & 0.68 & 0.67 & 14.09 & 1.76 \\
Canada & 0.78 & 0.78 & 0.78 & 0.78 & 0.78 & 7.16 & 2.85 \\
Denmark & 0.73 & 0.73 & 0.73 & 0.74 & 0.74 & 14.56 & 2.33 \\
Finland & 0.64 & 0.65 & 0.65 & 0.69 & 0.68 & inf & 1.88 \\
France & 0.98 & 0.98 & 0.98 & 0.98 & 0.99 & inf & 47.13 \\
Germany & 0.80 & 0.80 & 0.80 & 0.80 & 0.80 & 29.89 & 3.16 \\
Ireland & 0.76 & 0.76 & 0.76 & 0.76 & 0.76 & 13.10 & 2.56 \\
Italy & 0.90 & 0.90 & 0.90 & 0.90 & 0.91 & inf & 7.55 \\
Japan & 0.83 & 0.83 & 0.84 & 0.84 & 0.84 & 14.84 & 4.03 \\
Netherlands & 0.73 & 0.74 & 0.74 & 0.75 & 0.75 & 11.52 & 2.40 \\
Norway & 0.63 & 0.64 & 0.64 & 0.64 & 0.64 & 5.79 & 1.56 \\
Spain & 0.87 & 0.87 & 0.88 & 0.88 & 0.89 & 73.65 & 5.81 \\
Sweden & 0.59 & 0.61 & 0.61 & 0.67 & 0.62 & 3.93 & 1.71 \\
UK & 0.60 & 0.61 & 0.61 & 0.62 & 0.61 & 12.25 & 1.44 \\
US & 0.67 & 0.67 & 0.67 & 0.68 & 0.68 & 2.57 & 1.80 \\
\hline \hline
\end{tabular}




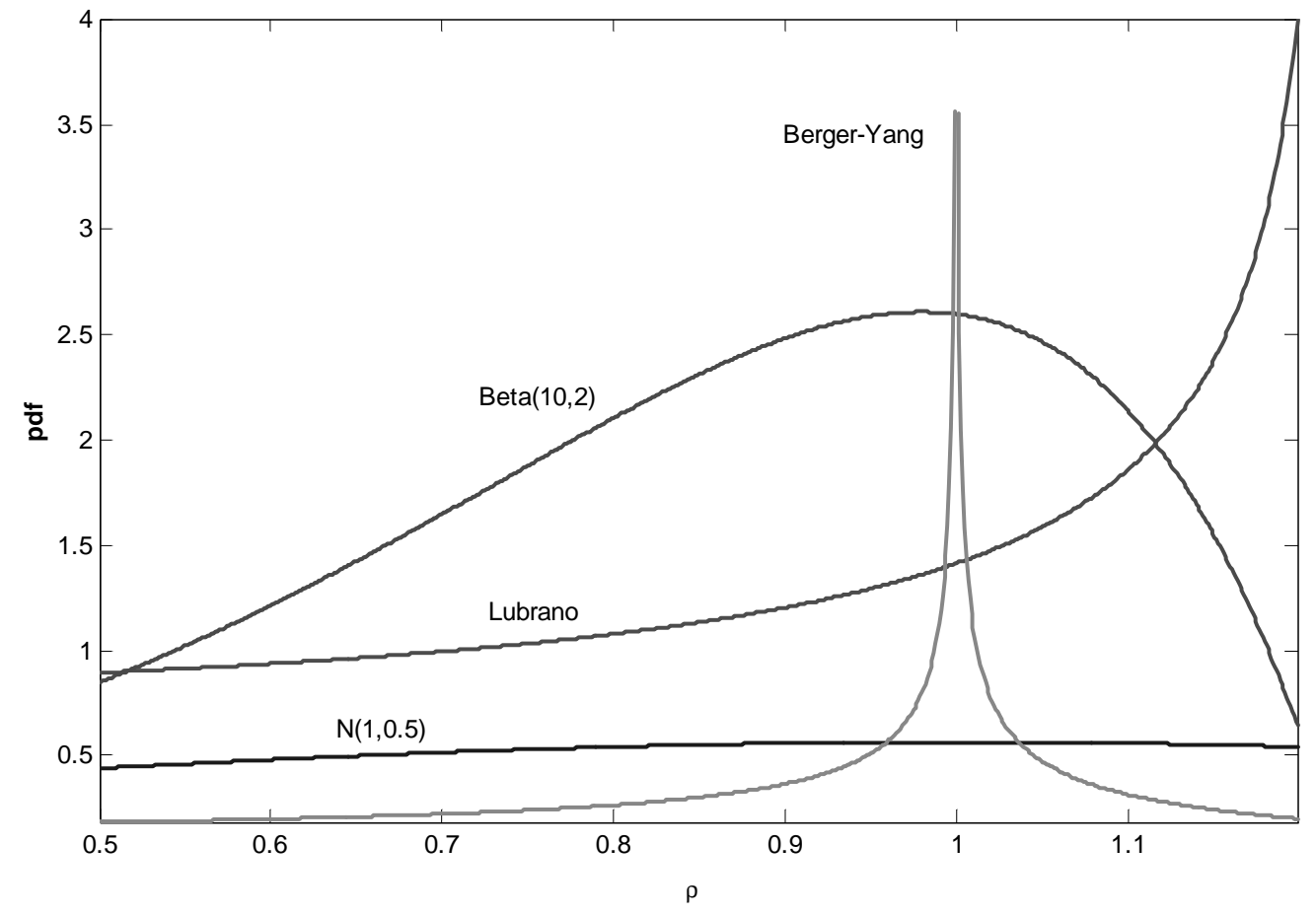

Figure 1: Prior distributions 